\section{Microsatellite Enrichment in Organisms with Large Genomes (Allium cepa L.)}

BioTechniques 24:796-802 (May 1998)

\begin{abstract}
To exploit the polymorphism of repeat numbers in short tandem repeat (STR) sequences (microsatellites) as molecular markers, STRs must be isolated and PCR primers must be developed in flanking sequences. In species with large genomes such as Allium cepa L. (onion and shallot), an efficient selection procedure for genomic fragments containing STRs is a crucial step. Here we describe a nonradioactive method for microsatellite isolation based on affinity capture of single-stranded restriction fragments annealed to biotinylated microsatellite oligonucleotides $(C A)_{10},(G A A)_{8}$
\end{abstract}

and $(A A C)_{8}$ followed by adapter-mediated genomic PCR. Cloning of the products in $\mathrm{E}$. coli and plasmid sequencing revealed more than $60 \%$ positive clones. Primers were designed in STR-flanking regions, and one or two bands were amplified in 13 diploid onion and five shallot accessions. Allelism of the bands was confirmed by product sequencing.

\section{INTRODUCTION}

Short tandem repeats (STR), also referred to as microsatellites, are abundant sequences dispersed throughout most eukaryotic nuclear genomes. In plants, they have been shown to be polymorphic at an intraspecific level in several cases $(10,12,15)$. The isolation of microsatellites by standard techniques (screening plasmid or phage libraries by colony hybridization with radioactively labeled oligonucleotide probes) turned out to be a cumbersome and non-costeffective procedure. Polymerase chain reaction (PCR)-based enrichment of previously cloned inserts $(7,9)$, novel PCR-based techniques (e.g., Reference 4) and techniques based on hybridization selection of restriction fragments before cloning $(2,6)$ have proven to be more powerful tools in microsatellite marker development in both plants and animals. The first approach to obtain microsatellite sequences in onion by hybridizing immobilized DNA from random-amplified polymorphic DNA (RAPD) gels to microsatellite oligonucleotides (3) and sequencing of positive bands failed. Microsatellite enrichment using microsatellite oligonucleotides cross-linked to nylon filter pieces as a probe, according to Reference 2, was not successful in onion.

Here we describe the nonradioactive establishment of a genomic RsaI library highly enriched for three micro- 
satellite motifs- $(\mathrm{CA})_{\mathrm{n}},(\mathrm{GAA})_{\mathrm{n}}$ and $(\mathrm{CAA})_{\mathrm{n}}-$ known to exist in the onion genome (13).

\section{MATERIALS AND METHODS}

Chromatin was extracted (14) from leaves of vegetatively propagated plants of a common onion ('Kaba', accession
ALL0917; Genebank Gatersleben, Germany). DNA was isolated from this chromatin fraction using a standard cetyltrimethylammonium bromide $(\mathrm{CTAB})$ procedure and subsequently purified from residual polysaccharides and RNA (11). Six micrograms of genomic DNA were digested by bluntend-generating restriction endonucle-

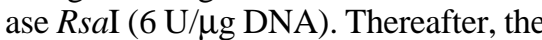

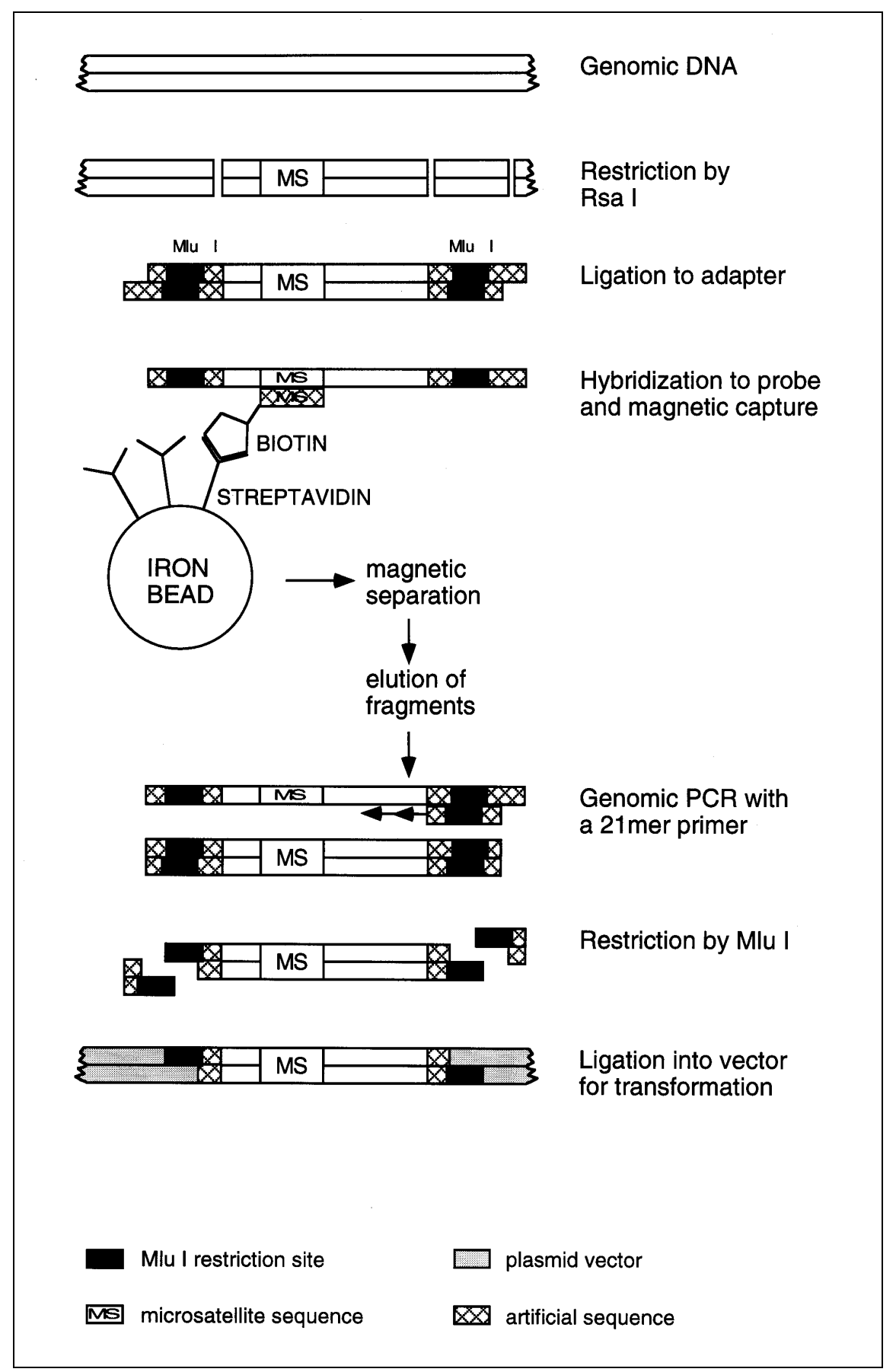

Figure 1. Schematic representation of the microsatellite enrichment method described in the text. 


\section{Short Technical Reports}

hybrid oligonucleotides from a 5 -phosphorylated 25-mer and a 21-mer with overlapping complementary sequence containing an MluI site were ligated to the blunt termini of the restriction fragments using $50 \mathrm{ng}$ adapter-to-primer hybrid per $1 \mu \mathrm{g}$ of genomic DNA (2).

Restriction by MluI and subsequent ligation were performed in one tube at $37^{\circ} \mathrm{C}$ using $0.5 \times$ universal potassium glutamate buffer (8). After $2 \mathrm{~h}, 1 \mathrm{U}$ of T4 DNA Ligase (Amersham Pharmacia Biotech, Little Chalfont, Bucks, Eng- land, UK) per $\mu \mathrm{g}$ DNA was added, followed by an additional 2-h incubation at $37^{\circ} \mathrm{C}$ to allow recutting of restriction fragment multimers generated during ligation. The constructs were then heatdenatured and allowed to hybridize to biotinylated microsatellite oligonucleotides (150 nM solution of each oligonucleotide, $6 \times$ standard saline citrate $[\mathrm{SSC}])$ of sequence types listed above $(6,13)$. These hybrids were subsequently bound to streptavidin-coated magnetic beads (Dynabeads ${ }^{\circledR}$; Dynal

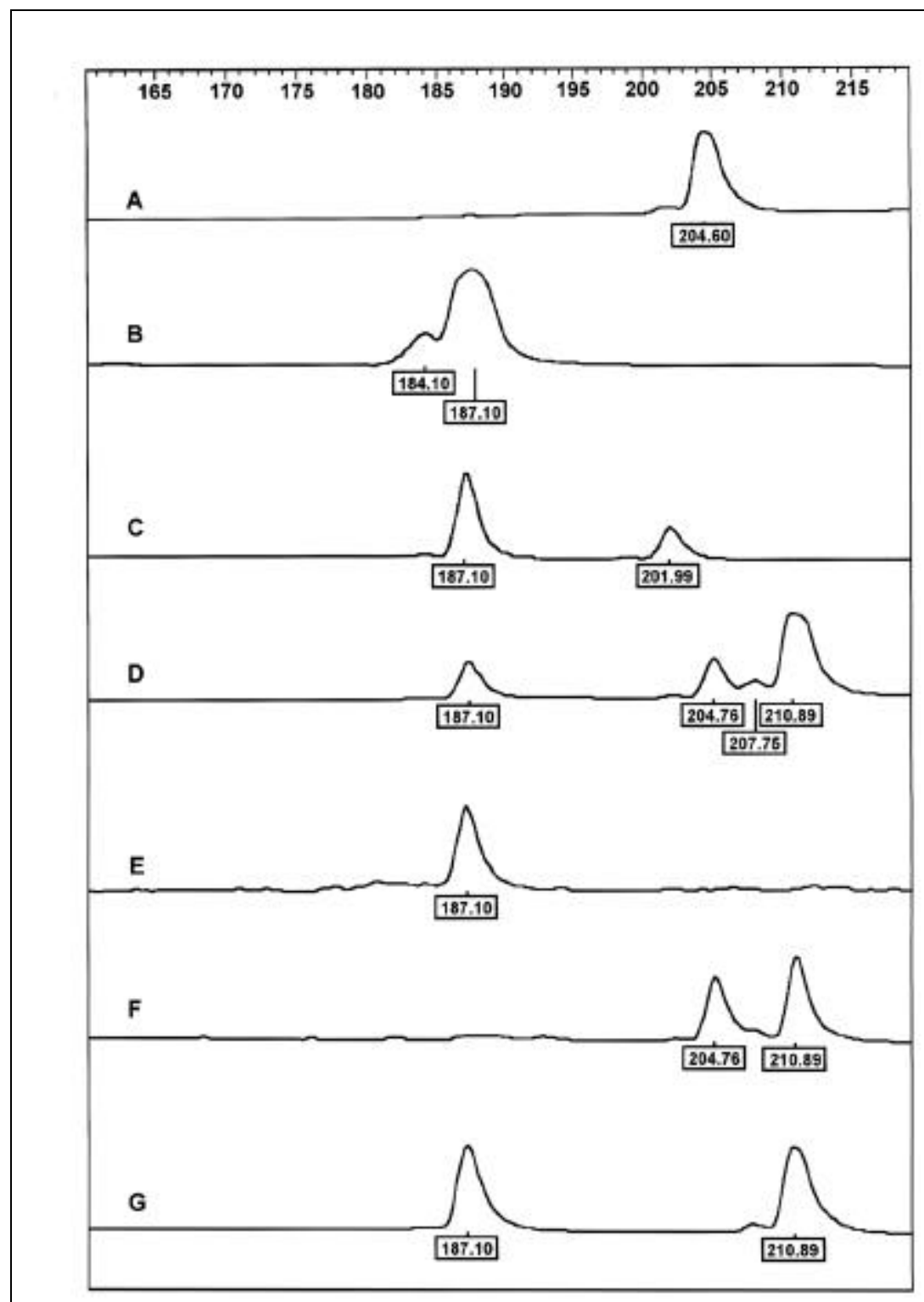

Figure 2. Seven fluorograms showing electrophoretic analysis of PCR fragments obtained with one primer fluorescently labeled at microsatellite locus AMS08 in onions (Allium cepa L.). (A-E) Common onions. (F and G) Shallots. (A) Accession 'KaBa', ALL0917 from which microsatellite loci have been isolated. Fragment sizes given in bp.

GmbH, Hamburg, Germany) (350 $\mu \mathrm{g}$ beads in $100 \mu \mathrm{L} 6 \times$ SSC final vol). Beads were then washed twice at lowstringency conditions $\left(25^{\circ} \mathrm{C}\right.$ in $2 \times \mathrm{SSC}$, $0.1 \%$ sodium dodecyl sulfate [SDS], 5 min each), four times at high-stringency conditions (twice in $1 \times$ SSC at $25^{\circ} \mathrm{C}, 5$ min each, and twice in $1 \times \mathrm{SSC}$ at melting temperature $\left[\mathrm{T}_{\mathrm{m}}\right]-5^{\circ} \mathrm{C}$ for 2 and $5 \mathrm{~min}$, respectively) and eluted as single-stranded fragments in triple-distilled water.

The obtained DNA solution $(0.2-1.0$ $\mu \mathrm{L})$ served as template for PCR $(28-30$ cycles with $56^{\circ} \mathrm{C}$ annealing temperature) using the 21-mer oligonucleotide as primer. The amplification products were MluI-digested to obtain vectorcompatible, sticky-ended fragments. Restriction fragments selected in this way were ligated in the presence of $M l u \mathrm{I}$ into a plasmid vector (pCRScript $^{\mathrm{TM}} \mathrm{Amp}^{+}$; Stratagene, La Jolla, CA, USA), which had been linearized with BssHII and dephosphorylated, thus creating a site that cannot be recut by $M l u \mathrm{I}$ (Figure 1). This was necessary to prevent religation of the inserts. T4 DNA Ligase and MluI (both from Amersham Pharmacia Biotech) performed together very well in $0.5 \times$ potassium glutamate buffer (8).

The ligated vector fragments were transformed into chemically competent E. coli XL1-Blue Strain Cells (Stratagene) and plated onto LB agar containing $2.67 \times 10^{-4} \mathrm{M}$ isopropyl $\beta$-D-thiogalactopyranoside (IPTG), $3.18 \times 10^{-4}$ M 5-bromo-4-chloro-3-indolyl- $\beta$-D-galactopyranoside (X-gal) and $30 \mathrm{mg} / \mathrm{L}$ carbenicillin. Plasmid DNA was isolated from white colonies using the QIAGEN ${ }^{\circledR}$ Plasmid Mini Kit (Qiagen GmbH, Hilden, Germany). Colony PCR was performed under reported PCR conditions (16) using cells from single colonies adhering to a $200-\mu \mathrm{L}$ pipet tip washed into $30 \mu \mathrm{L}$ of master mixture.

Most of the recombinant clones contained inserts of suitable length (from about 400 up to $1000 \mathrm{bp}$ ) and were sequenced on a Model 377 Fluorescent Sequencer (PE Applied Biosystems, Foster City, CA, USA) without further screening. Primer pairs were designed in the microsatellite flanking regions using the DesignerPCR ${ }^{\mathrm{TM}}$ computer program (Research Genetics, Huntsville, AL, USA), and PCR was per- 
formed with these primers, one of each being 5'-fluorescein-labeled, using genomic DNA from 14 onions and five shallots as templates. PCR products were checked on 3\% agarose gels (45 $\mathrm{mM}$ Tris-borate, $1 \mathrm{mM}$ EDTA) and then analyzed on a Model 377 Fluorescent Sequencer (15-cm electrophoresis glass plates) following the protocol provided by the manufacturer. Resulting data were processed with GENESCAN ${ }^{\mathrm{TM}}$ and GENOTYPER ${ }^{\text {TM }}$ Fragment Analysis Software (PE Applied Biosystems).

\section{RESULTS}

Twenty-nine of forty-eight readable sequences contained microsatellites, in most cases corresponding to the oligonucleotides used as probes. No duplicate sequences were observed. This indicates a high degree of microsatellite enrichment. So far, primer pairs in the flanking regions of 16 microsatellite loci (AMS01-AMS16) have been developed, six of which amplify one or two bands from the diploid genomes in 18 of the 19 onion genotypes. Using fluorescent fragment analysis, six alleles (showing sizes of 184, 187, 202, 205, 208 and $211 \mathrm{bp}$ ) could be distinguished at locus AMS08 in the 19 genotypes mentioned above (examples shown in Figure 2).

Sequencing some of the AMS08 amplification products showed that the length differences were due to various numbers of a trinucleotide repeat. These primer pairs therefore reveal polymorphic single-locus genotypes.

\section{DISCUSSION}

We have obtained polymorphic, codominant, single-locus markers with our approach. Homology between pu- tative alleles was verified by spot-check sequencing. As an additional advantage, some of the expense and timeconsuming procedures of conventional microsatellite marker development could be avoided (e.g., radioisotopes, large amounts of probe oligonucleotides and hybridization screening). The onion genome consists of $17.4 \times 10^{9} \mathrm{bp}$ (1). Studies on large genomes giving spurious hybridization signals with microsatellites raise the necessity of new methods in marker development (5, 6,9). The method of Kandpal et al. (5) requires PCR before enrichment selection, which initially reduces the population of fragments, whereas the method of Ostrander et al. (9) starts with bacterial cloning, which hampers selection by elimination or at least shortening of microsatellite loci during bacterial growth. Enrichment methods based on immobilization and fixation of genomic fragments using oligonucleotide probes (2) turned out to be difficult to optimize. Calculated hybridization temperatures often failed, probably because there is no control of the apparent probe length actually available for hybridization. For instance, a cross-linked (CT) $)_{12}$ probe may contribute between 0 and 10 core motifs to hybridization, which means a theoretical hybridization temperature interval between $<15^{\circ}$ and $77^{\circ} \mathrm{C}$. This makes a stringent selection much more difficult than the selection by annealing in liquid medium and subsequent retention by the high-affinity mechanism of streptavidin-biotin bonding. Independence of microsatellite selection and capture of the complex improves control over selection parameters without major influence on the yield. Previously published methods achieved a maximum enrichment rate of $50 \%$, whereas our approach achieves a slightly higher efficiency $(>60 \%)$ with a simpler procedure.

\section{REFERENCES}

1.Bennett, M.D. and J.B. Smith. 1976. Nuclear DNA amounts in angiosperms. Philos. Trans. R. Soc. Lond. 274:227-274.

2.Edwards, K.J., J.H.A. Barker, A. Daly, C. Jones and A. Karp. 1996. Microsatellite libraries enriched for several microsatellite sequences in plants. BioTechniques 20:758760 .

3.Ender, A., K. Schwenk, T. Städler, B. Streit 
and B. Schierwater. 1996. RAPD identification of microsatellites in Daphnia. Mol. Ecol. 5:437-441.

4.Fisher, P.J, R.C. Gardner and T.E. Richardson. 1996. Single locus microsatellites isolated using 5' anchored PCR. Nucleic. Acids. Res. 24:4369-4371.

5.Kandpal, R.P., G. Kandpal and S.M. Weissman. 1994. Construction of libraries enriched for sequence repeats and jumping clones, and hybridization selection for region specific markers. Proc. Natl. Acad. Sci. USA 91:88-92.

6.Kijas, J.M.H., J.C.S. Fowler, C.A. Garbett and M.R. Thomas. 1994. Enrichment of microsatellites from the citrus genome using biotinylated oligonucleotide sequences bound to streptavidin-coated magnetic particles. BioTechniques 16:656-662.

7.Lench, N.J., A. Norris, A. Bailey, A. Booth and A.F. Markham. 1996. Vectorette PCR isolation of microsatellite repeat sequences using anchored dinucleotide repeat primers. Nucleic. Acids. Res. 24:2190-2191.

8.McClelland, M., J. Hanish, M. Nelson and Y. Patel. 1988. KGB: a single buffer for all restriction endonucleases. Nucleic Acids Res. 16:364.

9.Ostrander, E.A., P.M. Jong, J. Rine and G.
Duyk. 1992. Construction of small-insert genomic libraries highly enriched for microsatellite repeat sequences. Proc. Natl. Acad. Sci. USA 89:3419-3423.

10.Powell, W., M. Morgante, C. Andre, M. Hanafey, J. Vogel, S. Tingey and A. Rafalski. 1996. The comparison of RFLP, RAPD, AFLP and SSR (microsatellite) markers for germplasm analysis. Mol. Breed. 2:225-238.

11.Rether, B., G. Delmas and A. Laouedj. 1993. Polysaccharide-free DNA from plants. Plant. Mol. Biol. Rep. 11:333-337.

12.Saghai-Maroof, M.A., R.M. Biyashev, G.P. Yang, Q. Zhang and R.W. Allard. 1994. Extraordinarily polymorphic microsatellite DNA in barley: species diversity, chromosomal locations, and population dynamics. Proc. Natl. Acad. Sci. USA 91:5466-5470.

13.Sharon, D., A. Adato, S. Mhameed, U. Lavi, J. Hillel, M. Gomolka, C. Epplen and J.T. Epplen. 1995. DNA fingerprints in plants using simple sequence repeat and minisatellite probes. Hort. Sci. 30:109-112.

14.Steinmüller, K. and K. Apel. 1986. A simple and efficient procedure for isolating plant chromatin which is suitable for studies of DNAase I-sensitive domains and hypersensitive sites. Plant Mol. Biol. 7:87-94.

15.Weising, K., R.W.M. Fung, D.J. Keeling,
R.G. Atkinson and R.C. Gardner. 1996. Characterization of microsatellites from $A c$ tinidia chinensis. Mol. Breed. 2:117-131.

16.Zeng, L.-W. and M. Kreitman. 1996. Simple strategy for sequencing cDNA clones. BioTechniques 21:446-452.

Work of the authors is funded by the European Commission, FAIR Project No. CT95-0465. Address correspondence to $\mathrm{Dr}$. Konrad Bachmann, Taxonomy Department, Institute of Plant Genetics and Crop Plant Research (IPK), Corrensstrasse, 3, D06466 Gatersleben, Germany. Internet: bachmann@ipk-gatersleben.de

Received 1 December 1997; accepted 2 February 1998.
Dirk Fischer and Konrad
Bachmann
Institute of Plant Genetics and
Crop Plant Research
Gatersleben, Germany 